\title{
Ultrastructure of gap junction and Cx43 expression in gastric cancer tissues of the patients
}

\author{
Chun-Hui Li, Mei-Ling Hao, Yu Sun, Zhu-Jun Wang, Jian-Ling Li
}

Department of Pathology, The Affiliated Hospital of Chengde Medical College, Chengde, Hebei, China

Submitted: 28 April 2017

Accepted: 21 August 2017

Arch Med Sci 2020; 16 (2): 352-358

DOI: https://doi.org/10.5114/aoms.2020.92859

Copyright $\Subset 2020$ Termedia \& Banach

\section{Abstract}

Introduction: Gap junctions are intercellular channels formed by connexin facilitating communication between cells by allowing transfer of ions and small signaling molecules. Connexin 43 (Cx43) is the most ubiquitous connexin in human tissues. Ample evidence suggests the role of gap junction and its connexins such as connexin 43 in human cancers including gastric cancer, which has an important place in the worldwide incidence of cancer and cancer-related deaths. Due to a number of contradictory studies and insufficient detailed examination in specific cancers, such as gastric cancer, more data on the role of gap junctions and their connexins such as Cx43 involved in gastric cancer remain necessary.

Material and methods: Transmission electron microscopy, Western blotting and RT-PCR were used to show the ultrastructure damage of the gap junction in the gastric carcinoma tissue as well as the expression of $C \times 43$ protein and mRNA, respectively.

Results: Ultrastructure damage of the gap junction in gastric carcinoma tissue was shown while poorly differentiated tissue experienced greater damage. The expression of $\mathrm{C} x 43$ protein and mRNA was higher in healthy gastric tissue than in carcinomatous gastric tissue $(p<0.05)$. There was higher expression of $\mathrm{C} \times 43$ protein and mRNA in high-medium differentiation than in poor differentiation $(p<0.05)$. Cx43 protein and mRNA expression is not statistically significant for different ages and sex (such as for $>56$ and $\leq 56$ years) $(p>0.05)$.

Conclusions: Ultrastructural changes of gap junctions with abnormal $\mathrm{C} \times 43$ expression are associated with occurrence and development of gastric cancer, which provides a new research direction for gastric cancer pathogenesis and targeted therapy.

Key words: gastric carcinoma, gap junction, $\mathrm{C} \times 43$, ultrastructure.

\section{Introduction}

Intercellular communication is an important mechanism involved in physiological processes and associated with many diseases and injuries [1]. In multicellular organisms, individual cells communicate with each other to exchange their nutrients and signals, which are necessary for their biological processes such as proliferation, differentiation, apoptosis, wound healing, and homeostasis for maintenance of the organization. There are multiple strategies multicellular organisms have evolved, such as neural or endocrine systems which mediate long-range interactions or direct physical or cell-cell contact which mediate short-range inter-

\author{
Corresponding author: \\ Chun-Hui Li \\ Department of Pathology \\ The Affiliated Hospital \\ of Chengde Medical College \\ Chengde 067000 \\ Hebei, China \\ E-mail: chli612@126.com
}


actions. Besides the field that was solely defined previously by the simple exchange of endocrine, autocrine and epicrine agents, some more complex forms of intercellular communication have been witnessed in the last few years that include microvesicles of various types, including exosomes, bioelectrical mechanisms, including local field potentials, volume transmission and special communication cells known as telocytes [2].

The gap junctions formed by connexin proteins facilitate intercellular communication between neighboring cells by allowing the transfer of ions and small signaling molecules [3]. Gap junctions are intercellular channels formed by connexins, a family of transmembrane proteins with the capacity to form gap junctions, and by the gap junctions, the cytoplasm of adjacent cells is directly linked. The most ubiquitously expressed connexin isoform in human tissues is connexin 43 (Cx43) [4]. With their important roles in maintaining the cell functions, dysregulations of connexin expression and function are involved in numerous pathogeneses, including cancer. It is evidenced that disorder in gap junctions and their structural subunits, connexins, often exists in, and sometimes causes, various proliferative disorders, including cancer [5].

Cancer has long been a major public health problem worldwide and is the second leading cause of death in the United States, where 1,685,210 new cancer cases and 595,690 cancer deaths were projected to occur in 2016 [6]. In China, with increasing incidence and mortality, cancer has become the leading cause of death and it was estimated that 4,292,000 new cancer cases and 2,814,000 cancer deaths would occur in 2015 in China [7]. Cancer is associated with many concerns and gastric cancer has an important place in the worldwide incidence of cancer and cancer-related deaths [8-13]. Gastric cancer is the fifth most common cancer and third leading cause of cancer-related death worldwide [14]. It was projected that there would be 26,370 new gastric cancer cases and 10,730 gastric cancer deaths in the United States in 2016 [6]. In China, 6,791,00 new gastric cancer cases and 498,000 gastric cancer deaths are expected [7]. Although ample evidence suggests the role of the gap junction and its connexins such as connexin 43 in a variety of human cancers, detailed examination in specific cancers, such as gastric cancer, is still not abundant; also, a number of contradictory studies have reported both pro- and anti-proliferative effects that often depend on the cell or tissue type or the microenvironment [5]. More data on the role of the gap junction and its connexins such as Cx43 involved in gastric cancer remain necessary.

This study investigated the changes in gap junction ultrastructure and $\mathrm{Cx} 43$ in normal gastric tissue and in carcinomatous gastric tissue at different degrees of differentiation in order to provide a novel research direction for gastric cancer pathogenesis as well as to provide potential targets for drug treatment.

\section{Material and methods}

\section{Objects}

Sixty-four samples of gastric carcinomatous tissue were surgically removed for collection from the patients in the affiliated hospital of Chengde Medical College from July 2010 to February 2013. In addition, 29 samples of normal gastric tissue were also collected (normal tissue was located more than $10 \mathrm{~cm}$ away from the carcinomatous edge). All of the samples were collected after formal written consent was obtained, in accordance with the guidelines of the Ethics Committee of the Affiliated Hospital of Chengde Medical College. None of the specimens had been treated with preoperative chemotherapy or radiotherapy. Among the 64 gastric carcinomatous samples, 47 samples were from male patients, and 17 samples were from female patients. The patients' age range was 45-67 years, with a median age of $56.5 \pm 10$.0. Twenty-six samples were of well-moderately differentiated adenocarcinoma, and 38 samples were of poorly differentiated adenocarcinoma. Twenty-seven of the samples did not have lymph node metastasis, whereas 37 cases did have lymph note metastasis. The TNM stage I and TNM stage II were merged as one group, while TNM stage III and TNM stage IV were merged as another group, thus it is kept consistent with the Figure 1. Fifty of the samples had serious penetration of carcinoma tissue, and 14 cases did not have serious penetration as detected by transmission electron microscopy, Western blotting, and RT-PCR.

\section{Reagents and instruments}

The reagents for preparing the electron microscopy sample were as follows: glutaraldehyde, osmic acid, Epon812, DDSA, uranium acetate, lead citrate, and so on. These were purchased from Sigma Aldrich in the United States. The Western blotting detection reagents and anti-Cx43 rabbit anti-human primary antibody were purchased from Bioworld Technology, Co, Ltd., Nanjing, China. RIPA organization cracking liquid (BB120031) was purchased from the Shanghai Bestbio Company (Bestbio, Shanghai, China). The BCA protein concentration determination kit, HRP-labeling sheep anti-rabbit secondary antibodies, and hypersensitive ECL luminous fluid were all purchased from the Beijing Solarbio Science and Technology Co., Ltd (Beijing, China). The remaining reagents (such as 30\% acrylamide, $\beta$-mercap- 
toethanol, $10 \%$ SDS, $10 \times$ ponceau S, $10 \%$ ammonium persulfate, $1.5 \mathrm{M} / 1.0 \mathrm{M}$ Tris- $\mathrm{HCl}$, TBST, $1 \times$ electrophoresis buffer and transmembrane liquid, developing powder, fixing powder, etc.) were from the Chengde Medical College Foundation Institute of Molecular Laboratory. The RT-PCR kit and DNA marker were purchased from the Dalian Treasure Biological Engineering Co., Ltd (Dalian, China). The PCR primers were designed and synthesized by the Beijing Parkson Gene Technology Co., Ltd. (Beijing, China). The primer sequences of $\mathrm{C} \times 43$ are 5'-TCTCGCCTATGTCTCCTCCTGG-3' (upstream primer) and 5'-AGTTAGAGATGGTGCTTCCCGC-3' (downstream primer), with amplified fragments of $156 \mathrm{bp}$. The primer sequences of P85 $\alpha$ are 5'-TGCTATGCCTGCTCTGTAGTGGT-3' (upstream primer) and 5'-GTGTGACATTGAGGGAGTCGTTG-3' (downstream primer), with amplified fragments of $175 \mathrm{bp}$. The primer sequences of $\beta$-actin are 5'-AGCGGGAAATCGTGCGTGAC-3' (upstream primer) and 5'-ACATCTGCTGGAAGGTGGAC-3' (downstream primer), with amplified fragments of $453 \mathrm{bp}$. The following instruments were used in this study: JEM-1200EX TEM (JEOL Ltd., Tokyo, Japan), LELCA ULTRACUT UCT Ultra microtome (LELCA, Vienna, Austria), ultraviolet spectrophotometry (DU800; Beckman, Brea, USA), enzyme standard instrument MK3 (Thermo, Waltham, USA), voltage steady flow electrophoresis apparatus (Shanghai Xin Industrial Co., LTD., Shanghai, China), PTC-220-PCR amplification (MJ Research, Inc., Watertown, USA), and 2020 D fluorescent UV digital imager (GoldSpring, Beijing, China).

\section{Transmission electron microscopy (TEM) assay}

Tissue samples were sliced into three 1-mm tissue blocks and fixed in $2.5 \%$ glutaraldehyde. Then, the samples were dehydrated step by step and embedded in Epon for ultrastructure observation by TEM technology.

\section{Western blotting assay}

Tissue were extracted with lysis buffer $(150 \mathrm{mM}$ $\mathrm{NaCl}, 1 \% \mathrm{NP}-40,0.1 \% \mathrm{SDS}, 2 \mathrm{mg} / \mathrm{ml}$ aprotinin and $1 \mathrm{mM}$ PMSF (Solarbio, Shijiazhuang, China)) for $30 \mathrm{~min}$ at $4^{\circ} \mathrm{C}$. Extracts were centrifuged at $15,000 \mathrm{~g}$ for $15 \mathrm{~min}$ at $4^{\circ} \mathrm{C}$. Supernatants containing total protein were then harvested. Aliquots, each containing $50 \mathrm{mg}$ of protein, were separated by $12.5 \%$ SDS-PAGE and transferred to PVDF membranes at $80 \mathrm{~V}$ or $120 \mathrm{~V}$ for $2 \mathrm{~h}$ at low temperature. The membranes were blocked in $5 \%$ skim milk for $2 \mathrm{~h}$, and proteins were detected using monoclonal antibodies overnight at $4^{\circ} \mathrm{C}$. Proteins were visualized using anti-rabbit IgG conjugated with horse radish peroxidase (HRP) for $2 \mathrm{~h}$ at $28^{\circ} \mathrm{C}$, respectively. Proteins were detected using the ECL Western blotting detection reagents and using IPP image analysis software for analysis of band density. The relative protein levels were calculated based on GAPDH as the loading control.

\section{RT-PCR assay}

Total RNA was extracted from gastric cancer tissues and adjacent normal tissues using a Trizol kit. The RT-PCR conditions for Cx43 were as follows: 35 cycles of $94^{\circ} \mathrm{C}$ pre-denaturation for $5 \mathrm{~min}, 94^{\circ} \mathrm{C}$ denaturation reaction for $30 \mathrm{~s}, 58^{\circ} \mathrm{C}$ annealing reaction for $30 \mathrm{~s}$, and $72^{\circ} \mathrm{C}$ extension reaction for $1 \mathrm{~s}$ followed by a $72^{\circ} \mathrm{C}$ extension reaction for $5 \mathrm{~min}$. The RT-PCR conditions for $\beta$-actin were as follows: 30 cycles of $94^{\circ} \mathrm{C}$ pre-denaturation reaction for $5 \mathrm{~min}, 94^{\circ} \mathrm{C}$ denaturation reaction for $30 \mathrm{~s}, 55^{\circ} \mathrm{C}$ annealing reaction for $30 \mathrm{~s}$, and $72^{\circ} \mathrm{C}$ extension reaction for $1 \mathrm{~min}$ followed by a $72^{\circ} \mathrm{C}$ extension reaction for $5 \mathrm{~min}$. Then, $2 \%$ agarose gel electrophoresis was used for detecting and quantifying the PCR-amplified products. Positive staining of the electrophoresis gel resulted in the appearance of the corresponding sites. $\beta$-actin was used as the internal standard, and the gel electrophoresis results were measured by an electrophoresis image analysis system.

\section{Statistical analysis}

SPSS17.0 was used for analyzing the experimental data. The gray value was expressed by means $\pm \mathrm{SD}$. An independent two-sample $t$-test was assumed for detection, and $p<0.05$ was defined as having statistical significance.

\section{Results}

\section{Ultrastructure of gap junction observed by electron microscopy}

In the normal gastric tissue intercellular junction in the glandular cavity, tight junctions, desmosomes, and gap junctions were observed. The intercellular junction structure was damaged in the well-moderately differentiated gastric cancer tissues and was seriously damaged in the poorly differentiated gastric carcinoma (Figure 2).

\section{Expression of $\mathrm{C} \times 43$ protein detected by Western blotting}

The relative $\mathrm{C} \times 43$ protein content in the 29 cases of normal gastric tissue was $0.82 \pm 0.04$, and in the 64 cases of gastric cancer tissue, it was 0.51 \pm 0.11 . The differences are statistically significant $(p<0.05)$. The relative $\mathrm{C} \times 43$ protein content in the well-moderately differentiated gastric cancer tissues was $0.64 \pm 0.04$, whereas in the poorly differentiated gastric carcinoma, the content was 0.42 
\pm 0.06 . The relative content in TNM stage I/II was $0.67 \pm 0.03$, and it was $0.37 \pm 0.09$ in TNM stage III/IV. The relative $\mathrm{C} \times 43$ protein content in the group in which there was serious membrane penetration was $0.45 \pm 0.09$, and in the group without serious membrane penetration, it was $0.72 \pm 0.03$. The relative expression level of the $\mathrm{C} \times 43$ protein in the group with lymph node metastasis was 0.41 \pm 0.07 , and in the group without lymph node metastasis, it was $0.65 \pm 0.04$. In the independent two-samples $t$-test, the aforementioned differences had statistical significance $(p<0.05, p<0.05$, $p<0.05, p<0.05$ ), whereas the differences in the Cx43 protein expression level between different sex and different age groups were not statistically significant (both $p>0.05$, Figures 1,3 ).

\section{Expression of Cx43 mRNA detected by RT-PCR}

The relative $\mathrm{C} \times 43$ mRNA content in the 29 cases of normal gastric tissue was $0.73 \pm 0.07$, and in the 64 cases of gastric cancer tissue, the content was $0.40 \pm 0.07$. The differences are statistically significant $(p<0.05)$. The relative $\mathrm{C} \times 43$ mRNA content in the well-moderately differentiated gastric cancer tissues was $0.57 \pm 0.07$, whereas the relative content in the poorly differentiated gastric carcinoma was $0.28 \pm 0.05$. The relative content in TNM stage I/II was $0.52 \pm 0.06$, and the relative content in TNM stage III/IV was $0.29 \pm 0.05$. The relative Cx43 mRNA content in the group with serious membrane penetration was $0.34 \pm 0.06$, and in the group without serious membrane penetration, the
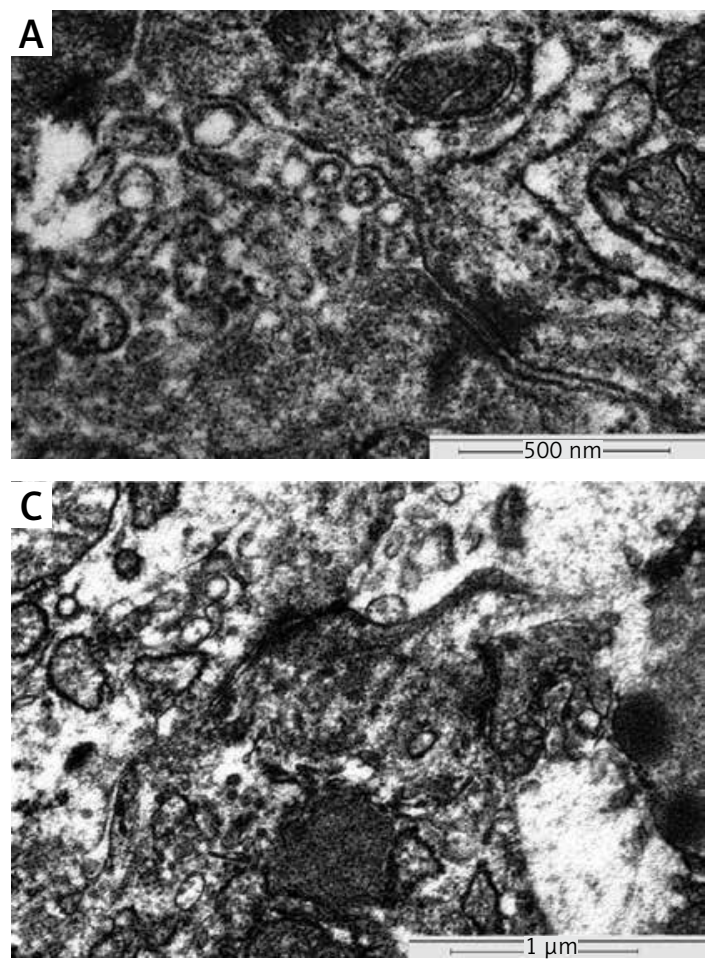

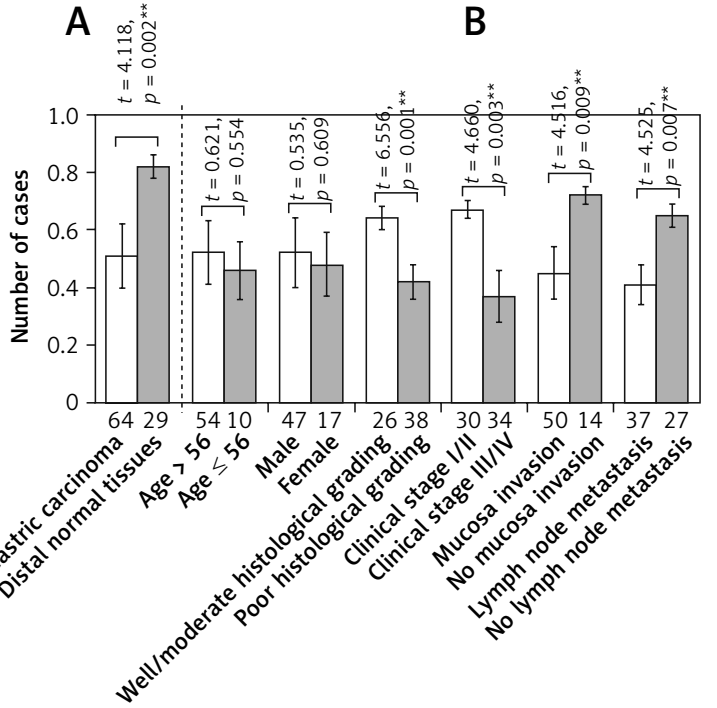

Figure 1. Level of $\mathrm{C} \times 43$ protein expression in normal and carcinomatous gastric tissues determined by western blot. Error bars indicate standard deviation of the means. Double asterisks indicate $t$-test $p<0.01$. A - Cx43 protein expression in normal and carcinomatous gastric tissues. B - Cx43 protein expression in carcinomatous gastric tissues with different clinicopathological features

content was $0.61 \pm 0.06$. The relative expression level of Cx43 mRNA in the group with lymph node metastasis was $0.32 \pm 0.05$, and in the group without lymph node metastasis, it was $0.51 \pm 0.06$. According to the independent two-samples $t$-test, the differences had statistical significance $(p<0.05, p$ $<0.05, p<0.05, p<0.05$ ); however, the differences in the Cx43 mRNA expression level between differ-

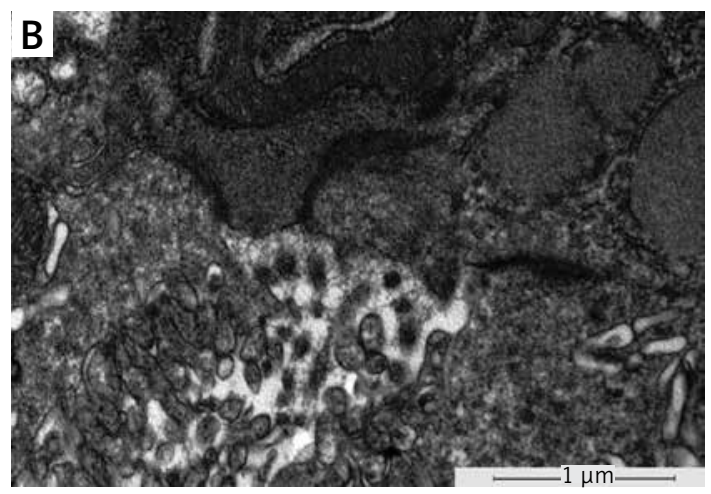

Figure 2. Ultrastructure of gap junction in normal gastric tissue, well-moderately differentiated and poorly differentiated gastric carcinoma tissue. The gap junction ultrastructure in the tissues was determined by electron microscopy. A - Complete gap junctions in normal gastric tissue (TEM 100000x, bar $=500 \mathrm{~nm}$ ). $\mathbf{B}$ - Incomplete gap junctions in well-moderately differentiated gastric carcinoma

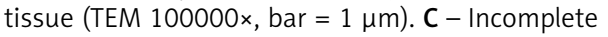
gap junctions in poorly differentiated gastric carcinoma tissue (TEM100000× , bar $=1 \mu \mathrm{m})$ 


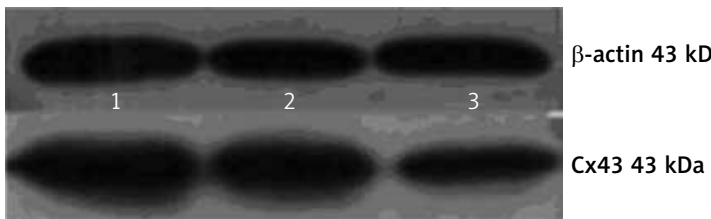

Figure 3. Lanes of Cx43 protein expressed in gastric cancer and normal gastric mucosa tissues determined by western blot. 1 - normal gastric mucosa, 2 - well and moderately differentiated gastric cancer, 3 - poorly differentiated gastric cancer

A
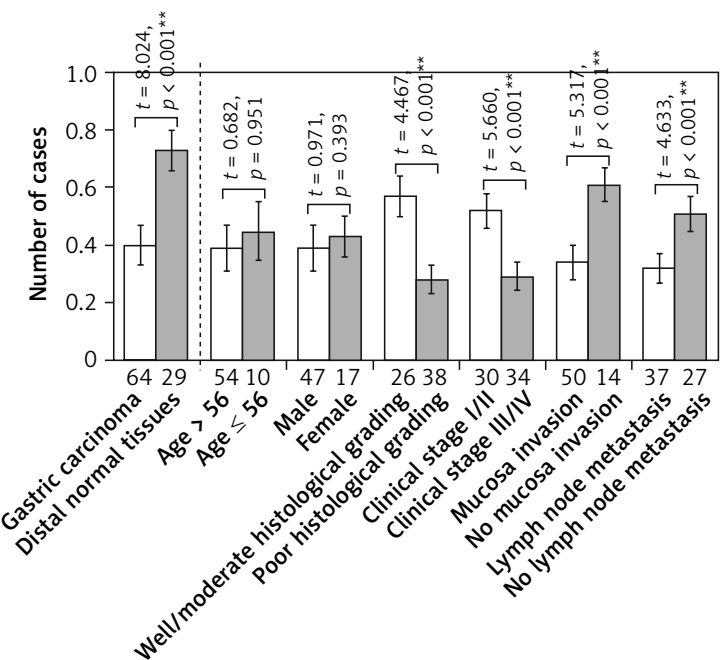

Figure 5. Level of Cx43 mRNA expression in normal and carcinomatous gastric tissues determined by RT-PCR. Error bars indicate standard deviation of the means. Double asterisks indicate $t$-test $p<0.01$. A - Cx43 mRNA expression in normal and carcinomatous gastric tissues. B - Cx43 mRNA expression in carcinomatous gastric tissues with different clinicopathological features

ent sex and different age groups were not statistically significant (both $p>0.05$, Figures 4,5 ).

\section{Discussion}

Gap junction intercellular communication (GIIC) is a means of communication for adjacent cells that is mediated by gap junctions. It is involved in the passing of information among cells and plays an important role in the regulation of cell proliferation and differentiation. The main channel component is the gap junction protein connexin 43 (Cx-43). At present, both domestic and foreign researchers are conducting research on the relationship between GIIC and cancer development. This is because a defect in Cx expression, a loss in GIC function, and intercellular communication disorders can result in the loss of normal contact between cells. As a result, regulatory signals cannot reach malignant cells from normal cells, and thus unlimited proliferation, material energy metabolic abnormalities, and other disorders result. These factors may contribute to tumor occurrence

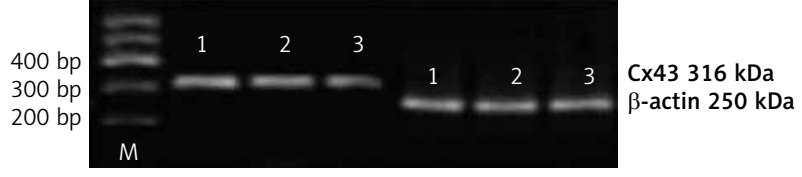

Figure 4. Lanes of Cx43 mRNA expressed in gastric cancer and normal gastric mucosa tissues determined by RT-PCR. 1 - Normal gastric mucosa, 2 - well and moderately differentiated gastric cancer, 3 - poorly differentiated gastric cancer

[15-18]. Due in part to this possibility, with the consideration that cancer, associated with many concerns, has long been a major public health problem worldwide [6, 14, 19-21], gap junctions and gap junction protein have become hot topics in current bioscience research. This study investigated the changes in gap junction ultrastructure and $\mathrm{Cx} 43$ in normal gastric tissue and in carcinomatous gastric tissue at different degrees of differentiation in order to provide a novel research direction for gastric cancer pathogenesis as well as to provide potential targets for drug treatment.

Direct information transfer between cells is achieved by intercellular junctions, which include gap junctions, desmosome junctions, and tight junctions. Tight junctions do not have communication capabilities, and there is little research on the communication capabilities of desmosome junctions. However, the biological function of gap junctions has recently received a great deal of attention. The gap junction is a kind of plate connection with a large contact area, and it is more common in the deep side of epithelial cells where the adjacent cell membrane is separated by $2 \mathrm{~nm}$. The adjacent cell membrane contains many hexagonal particles (6-8 $\mathrm{nm}$ in size) that are known as connexons. Each connexon is composed of six transmembrane intrinsic protein molecules, which form a central tunnel that is $2 \mathrm{~nm}$ in diameter. The tunnels of adjacent connexons on the plasma membranes of corresponding connected cells form direct channels between the cells. This tiny pipe-type channel is composed of six sub-units and the shifting of the six sub-units turns on or turns off the channel $[19,22]$. Through observing normal and cancerous gastric tissue ultrastructure via electron microscopy, we observed that the normal gastric mucosal gap junction structure is complete, whereas the gap junction structure of gastric cancer is damaged, and the poorly differentiated gastric cancer tissue's gap junction structure has a greater degree of damage. It can be concluded that because there is a lack of cell gap junction communication function, the GIIC function is reduced or suppressed after the cells have transformed. This may be an important mechanism for promoting carcinogenesis, and the lack of gap junctions may be involved in tumor occurrence. 
Gap junctions are the structural basis of cellular communication, and the diversity of gap junction function is based on the diversity of the molecular structure of gap junction proteins. One such membrane protein is $\mathrm{Cx} 43$, which is the structural basis of cell metabolism coupling and impulse conduction. Cx43 is an important member of the gap junction protein family and plays an important role in the intercellular information exchange and regulation of cell proliferation and differentiation in normal cells. Furthermore, it is also the main connecting protein in the GJIC of gastric cancer tissues [18].

Decreased or absent $\mathrm{C} \times 43$ expression is closely related to the occurrence and development of various tumors [23-30]. The inhibition of Cx43's effect on tumor growth is considered through the following: some growth inhibitors are distributed by gap junctions (GJ), such as through CAMP, to participate in GJ-dependent growth inhibition so that GIIC function is decreased or absent, and the control signals from normal cells cannot reach the malignant cells, and the resulting unrestricted growth can lead to tumor occurrence $[24,25,27,28]$. In normal cell carcinoma, the expression of the gap junction protein is decreased, and there is GIIC dysfunction, which inhibits the function of cellular communication and allows for abnormal growth of tumor cells. Thus, Cx43 plays an important role in tumor malignant progression. The finding of the study also confirmed that the protein expression of $\mathrm{C} \times 43$ in normal gastric mucosa tissues was higher than that in carcinoma tissues, which indirectly confirms that there was gap junction communication dysfunction in the process of cell carcinogenesis. Tang B $[28,31]$ and other researchers have found that the expression of $\mathrm{Cx} 43$ in gastric cancer cells is significantly lower than that in the adjacent normal cells. Moreover, the Cx43 expression in gastric cancer cells in the lymph nodes without metastasis was significantly higher than that of gastric cancer cells in the lymph nodes with metastasis. This suggests that low expression of Cx43 may contribute to the occurrence of gastric carcinoma.

In conclusion, this study confirmed that the expression of $\mathrm{Cx} 43$ in gastric cancer tissues was lower than that in normal gastric mucosa tissues, and the expression of Cx43 in gastric cancer with lymph node metastasis was lower than that without lymph node metastasis. Furthermore, the expression of $\mathrm{Cx} 43$ in gastric cancer in TNM stage I/II was higher than that in TNM stage III/IV. Therefore, we speculate that $\mathrm{C} \times 43$ may be involved in various tumor factors that affect the occurrence of gastric cancer and that $\mathrm{C} \times 43$ can affect the biological behavior of gastric cancer. As a result, decreased Cx43 expression levels and abnormal gap junction intercellular communication function may be involved in the progression of the malignant degree of gastric cancer and the metastasis of tumor cells.

\section{Acknowledgments}

This work was supported by a Hebei medical scientific research key project (No. ZL20140103).

\section{Conflict of interest}

The authors declare no conflict of interest.

\section{References}

1. Hussain MU. Connexins: the gap junction proteins. Anticancer Res 2015; 35: 1832.

2. Edelstein L, Fuxe K, Levin M, Popescu BO, Smythies J. Telocytes in their context with other intercellular communication agents. Semin Cell Dev Biol 2016; 55: 9-13.

3. Grek CL, Rhett JM, Bruce JS, Ghatnekar GS, Yeh ES. Connexin 43, breast cancer tumor suppressor: missed connections? Cancer Lett 2016; 374: 117-26.

4. Leithe E. Regulation of connexins by the ubiquitin system: implications for intercellular communication and cancer. Biochim Biophys Acta 2016; 1865: 133-46.

5. Aasen T. Connexins: junctional and non-junctional modulators of proliferation. Cell Tissue Res 2015; 360: 685-99.

6. Siegel RL, Miller KD, Jemal A. Cancer statistics, 2016. CA Cancer J Clin 2016; 66: 7-30.

7. Chen W, Zheng R, Baade PD, et al. Cancer statistics in China, 2015. CA Cancer J Clin 2016; 66: 115-32.

8. Mihmanli M, Ilhan E, Idiz UO, Alemdar A, Demir U. Recent developments and innovations in gastric cancer. World J Gastroenterol 2016; 22: 4307-20.

9. Fu J. Cx43 expressed on bone marrow stromal cells plays an essential role in multiple myeloma cell survival and drug resistance. Arch Med Sci 2017; 13: 236-45.

10. Woźniacki P, Skokowski J, Bartoszek K, Kosowska A, Kalinowski L, Jaśkiewicz J. The impact of the Polish mass breast cancer screening program on prognosis in the Pomeranian Province. Arch Med Sci 2017; 13: 441-7.

11. Kaliszewski K, Diakowska D, Strutyńska-Karpińska M, Wojtczak B, Domosławski P, Balcerzak W. Clinical and histopathological characteristics of patients with incidental and nonincidental thyroid cancer. Arch Med Sci 2017; 13: 390-5.

12. Jelski W, Laniewska-Dunaj M, Orywal K, Kochanowicz J, Rutkowski R, Szmitkowski M. The diagnostic value of alcohol dehydrogenase $(\mathrm{ADH})$ isoenzymes and aldehyde dehydrogenase (ALDH) measurement in the sera of patients with brain tumor. Arch Med Sci 2017; 13: 346-52.

13. Lu J, Sun LX, Lin ZB, et al. Antagonism by Ganoderma lucidum polysaccharides against the suppression by culturesupernatants of B16F10 melanoma cells on macrophage. Phytother Res 2014; 28: 200-6.

14. Lansdorp-Vogelaar I, Kuipers EJ. Screening for gastric cancer in Western countries. Gut 2016; 65: 543-4.

15. Li W, Hertzberg EL, Spray DC. Regulation of connexin43-protein binding in astrocytes in response to chemicalischemia hypoxia. J Biol Chem 2005; 80: 7941-8.

16. Ball KK, Harik L, Gandhi GK, et al. Reduced gap junctional communication among astrocytes in experimental diabetes: contributions of altered connexin protein levels 
and oxidative-nitrosative modifications. J Neurosci Res 2011; 89: 2052-67.

17. Giepmans BN. Role of connexin43-interacting proteins at gap junctions. Adv Cardiol 2006; 42: 41-56.

18. Fowler SL, Akins M, Zhou H, et al. The liver connexin32 interactome is a novel plasma membrane-mitochondrial signaling nexus. J Proteome Res 2013; 12: 2597-610.

19. Hoskovec D, Varga J, Dytrych P, Konecna E, Matek J. Peritoneal lavage examination as a prognostic tool in cases of gastric cancer. Arch Med Sci 2017; 13: 612-6.

20. Szynglarewicz B, Kasprzak P, Hałoń A, Matkowski R. Lobular carcinoma in situ of the breast - correlation between minimally invasivebiopsy and final pathology. Arch Med Sci 2017; 13: 617-23.

21. Nikolic I, Andjelkovic M, Zaric M, et al. Induction of mitochondrial apoptotic pathway by raloxifene and estrogen inhuman endometrial stromal ThESC cell line. Arch Med Sci 2017; 13: 293-301.

22. Sosinsky GE. Molecular organization of gap junction membrane channels. J Bioenerg Biomembr 1996; 28: 297-308.

23. Xu CX, Jia Y, Yang WB, et al. Relationship between Helicobacter pylori infection and expression of connexin $\mathrm{C} \times 32$ and $\mathrm{C} \times 43$ genes in gastric cancer and gastric precancerous lesions. Zhonghua Yi Xue Za Zhi 2008; 88: 1523-7.

24. Alasdair I, MacDonaid, Sun P, et al. A functional interaction between the MAGUK protein hD1g and the gap junction protein connexin43 in cervical tumour cells. Biochem J 2012; 446: 9-12.

25. Li J, Cheng L, Wang LJ, et al. Cell surface sialic acid inhibits $\mathrm{C} \times 43$ gap junction functions in constructed Hela cancer cells involving in sialylated $\mathrm{N}$-cadherin. Mol Cell Biochem 2010; 344: 241-51.

26. De Bock M, Kerrebrouck M, Wang N, et al. Neurological manifestations of oculodentodigital dysplasia: a Cx43 channelopathy of the central nervous system. Front Pharmacol 2013; 4: 120 .

27. Abrams CK, Scherer SS. Gap junctions in inherited human disorders of the central nervous system. Biochem Biophys Acta 2012; 1818: 2030-47.

28. Kim MS, Gloor GB, Bai D. The distribution and functional properties of Pelizaeus-Merzbacher-like disease-linked Cx47 mutations on Cx47/Cx47 homotypic and Cx47/ Cx43 heterotypic gap junctions. Biochem J 2013; 452: 249-58.

29. Sin WC, Crespin S, Mesnil M. Opposing roles of connexin43 in glioma progression. Biochim Biophys Acta 2012; 1818: 2058-67.

30. Plante I, Stewart MK, Barr K, et al. Cx43 suppresses mammary tumor metastasis to the lung in a $\mathrm{Cx} 43 \mathrm{mu}-$ tant mouse model of human disease. Oncogene 2011; 30: 1681-92

31. Tang B, Peng ZH, Yu PW, et al. Expression and significance of $\mathrm{Cx} 43$ and E-cadherin in gastric cancer and metastatic lymph nodes. Med Oncol 2011; 28: 502-8. 